
allemande

50-2 | 2018

Humanités environnementales - Quoi de neuf du côté des méthodes?

\title{
Prêcher à la fin de la Première Guerre mondiale (octobre-novembre 1918) : Albert Schweitzer
}

Matthieu Arnold

\section{(2) OpenEdition}

Journals

Édition électronique

URL : https://journals.openedition.org/allemagne/979

DOI : 10.4000 /allemagne.979

ISSN : 2605-7913

Éditeur

Société d'études allemandes

Édition imprimée

Date de publication : 30 décembre 2018

Pagination : 415-427

ISSN : 0035-0974

Référence électronique

Matthieu Arnold, «Prêcher à la fin de la Première Guerre mondiale (octobre-novembre 1918) : Albert Schweitzer ", Revue d'Allemagne et des pays de langue allemande [En ligne], 50-2 | 2018, mis en ligne le 30 décembre 2019, consulté le 18 mai 2021. URL : http://journals.openedition.org/allemagne/979 DOI : https://doi.org/10.4000/allemagne.979 


\title{
Prêcher à la fin de la Première Guerre mondiale (octobre-novembre 1918): Albert Schweitzer
}

\author{
- Matthieu Arnold *
}

Lorsque, à l'automne 1918, Albert Schweitzer prêche, en allemand, en l'église Saint-Nicolas (Strasbourg), il est un orateur expérimenté: il a débuté son activité de prédicateur vingt ans plus tôt, à la fin de la première partie de ses études de théologie, après avoir obtenu la «licentia concionandi» (autorisation de prêcher). De 1899 à 1912, il a prêché en tant que vicaire stagiaire puis, à partir du 14 novembre 1900, en tant que vicaire à la paroisse allemande de Saint-Nicolas ${ }^{(1)}$. Saint-Nicolas, qui avait la particularité de comporter aussi une paroisse française, était marquée par le libéralisme théologique: ses pasteurs examinaient de manière critique tant les dogmes que les textes bibliques. La prédication de Schweitzer se caractérise notamment par ses accents éthiques: à la suite de Jésus, dont il s'agit d’actualiser les préceptes, Schweitzer exhorte ses paroissiens à œuvrer à l'avènement du Royaume de Dieu, c'est-à-dire d'un monde qui soit de plus en plus imprégné de l'«Esprit de Dieu et de Jésus» ${ }^{(2)}$.

* Professeur en histoire moderne et contemporaine à la Faculté de théologie protestante, Université de Strasbourg, EA 4378.

1 De 1902 à 1912, Schweitzer a également été Privatdozent en Nouveau Testament à la Faculté de Théologie protestante de l'Université impériale de Strasbourg.

2 Les prédications de Schweitzer ont été éditées dans la série de ses «Werke aus dem Nachlaß»: Albert Schweitzer, Predigten 1898-1948, éd. Richard Brüllmann et Erich Grässer, Munich, Beck, 2001. - À l'exception de l'introduction de Richard BRÜLLMANN, «Der Prediger Albert Schweitzer» (op. cit., p. 25-57), les études consacrées aux sermons de Schweitzer sont peu nombreuses, et l'on nous permettra en conséquence de renvoyer à quelques-uns de nos propres travaux portant sur ce thème: Matthieu ARnold, Albert Schweitzer. Les années alsaciennes 1875-1913, Strasbourg, La Nuée Bleue, 2013 (2éd.), p. 65-113: «Le pasteur»; ID., «Les renvois autobiographiques dans les sermons de Lambaréné d'Albert Schweitzer», in: Dominique Dinet, François Igersheim (éd.), Terres d'Alsace, chemins de l'Europe. Mélanges offerts à Bernard Vogler, Strasbourg, Presses universitaires de Strasbourg, 2003, p. 33-49; ID., «Les animaux dans les sermons d'Albert Schweitzer», Revue d'Alsace, 132 (2006), p. 245-259; ID., "La mission dans les sermons d'Albert Schweitzer (1899-1909)», Positions luthériennes, 58 (2010), p. 51-69; ID., «La prédication d'Albert Schweitzer, vicaire à la paroisse de Saint-Nicolas (1898-1912)», Revue 
En mars 1913, fidèle à un projet qu'il avait caressé depuis l'automne de 1905, Schweitzer est parti comme médecin missionnaire pour l'Afrique, afin notamment d'expier les méfaits de la colonisation en soignant les indigènes et en leur annonçant le message de Jésus, qui libère de toutes les peurs ${ }^{(3)}$. Rapidement, il s'est délié de la promesse, faite le 11 mai à la Société des Missions de Paris, d'être «muet comme une carpe » ${ }^{(4)}$, et il a ajouté à son activité de médecin celle de prédicateur. Hélas, seuls quelques sermons de cette première période de son activité en Afrique nous ont été conservés ${ }^{(5)}$, et l'on ignore la teneur de sa prédication durant les années de guerre. Durant cette période, ses coreligionnaires restés en Alsace avaient, pour la majorité d'entre eux, sacrifié à la rhétorique guerrière et nationaliste qui caractérise la plupart des sermons de 1914$1918^{(6)}$, même si, sans doute, ces sermons alsaciens étaient moins outranciers que ceux qui avaient été prononcés dans le reste de l'Allemagne ${ }^{(7)}$.

En septembre 1917, Schweitzer et son épouse Hélène ont été rapatriés en Europe par les autorités françaises, en tant que prisonniers de guerre (ils étaient citoyens allemands, à l'œuvre au Congo français), puis internés successivement à Bordeaux, à Garaison (Hautes-Pyrénées) et à Saint-Rémy de Provence ${ }^{(8)}$. Cette expérience douloureuse marqua durablement les époux Schweitzer, même si Albert sut se rendre utile en tant que médecin ${ }^{(9)}$.

À l'été de 1918, les époux Schweitzer rentrèrent en Alsace. Le 18 août 1918, il prêcha à Gunsbach à l'occasion du $43^{\mathrm{e}}$ anniversaire de ministère pastoral de son père Louis Schweitzer. Deux semaines plus tard, le $1^{\text {er }}$ septembre, il se fit opérer d'un abcès à l'intestin. Le 13 octobre, il retrouva sa communauté de Saint-Nicolas : conformément à

d'Histoire et de Philosophie religieuses, 93 (2013), p. 377-395; ID., «Luther dans les sermons d'Albert Schweitzer (1899-1919)", Revue de l'Histoire du Protestantisme, 2 (2017), p. 157-167.

3 Voir Matthieu Arnold, «La mission dans les sermons d'Albert Schweitzer (1899-1909)», Positions luthériennes. Théologie, histoire, spiritualité, 58 (2010), p. 51-69.

4 Voir M. Arnold, Albert Schweitzer. Les années alsaciennes (note 2), p. 231.

5 Voir Albert Schweitzer, Les sermons de Lambaréné, éd. Jean-Paul Sorg et Philippe Aubert, Strasbourg, Oberlin, 2002.

6 Voir Matthieu Arnold, “Je ne suis pas venu pour apporter la paix...”. L'image et le message de Jésus-Christ dans les prédications de guerre, 1914-1918», in: Frédéric Rognon (dir.), Dire la guerre, penser la paix, Genève, Labor et Fides, 2014, p. 215-235; ID., "Les prédications de guerre protestantes prononcées en Alsace à l'occasion de l'anniversaire du Kaiser», Bulletin de la Société de l'Histoire du Protestantisme Français, 160 (2014), p. 57-76; ID., «Die Kriegspredigten in Elsass-Lothringen 19141918 », Jahrbuch für badische Kirchen- und Religionsgeschichte, 8-9 (2014-2015), p. 231-242.

7 Voir Wilhelm Pressel, Die Kriegspredigt 1914-1918 in der evangelischen Kirche Deutschlands, Göttingen, Vandenhoeck \& Ruprecht, 1967; Karl Hammer, Deutsche Kriegstheologie 1870-1918, Munich, Kösel-Verlag, 1971; Matthieu Arnold, «La chaire au service de la patrie: prédications protestantes françaises et allemandes durant la Première Guerre mondiale», Revue d'Allemagne et des pays de langue allemande, 36 (2004), p. 135-154.

8 Voir Albert Schweitzer, Ma vie et ma pensée (1931), Gunsbach, Éditions AISL, 2017, p. 191-199.

9 Voir Patti M. Marxsen, Helene Schweitzer. A Life of Her Own, s.1., Syracuse University Press, 2015, p. 50-58; Louis Schweitzer, Journal (1914-1919), Gunsbach - Munster, AISL - Société d'histoire du val et de la ville de Munster, 2015, p. 262 (7 février 1918) : "Carte de mon frère Auguste du 7 janvier. Il a reçu une longue lettre d'Albert et d'Hélène. Albert aurait pu résider au lieu de son choix, mais il a décidé de rester à Garaison où il peut s'occuper activement dans le domaine médical et apporter beaucoup d'aide aux malades qui y sont négligés.» 
la promesse que lui avait faite le Directoire de l'Église de la Confession d'Augsbourg en Alsace et en Lorraine en $1912^{(10)}$, il fut réintégré dans le corps pastoral (il était parti en Afrique en demandant un congé sans solde), mais à nouveau en tant que simple vicaire et non pas en tant que pasteur titulaire. Pourtant, son ancienne paroisse avait d'autant plus besoin de lui que l'un de ses collègues de la période 1902-1912, Charles Théodore Gérold, avait été suspendu de ses fonctions au début de la guerre par les autorités allemandes, et que l'autre pasteur titulaire, germanophile, venait de perdre son poste.

\section{Avenir de l'humanité et "respect de la vie»: les sermons d'Albert Schweitzer (13 octobre et 24 novembre 1918)}

Le 13 octobre 1918, cinq ans et demi après son départ pour l'Afrique, Schweitzer reprit son activité de prédicateur dans la paroisse qui lui était chère. Il prit pour texte de prédication Philippiens 4,7, passage biblique à partir duquel il avait délivré son message d'adieu le 9 mars 1913: «Der Friede Gottes, welcher höher ist denn alle Vernunft, bewahre eure Herzen und Sinne in Christo Jesu» (La paix de Dieu, qui surpasse toute intelligence, maintienne vos cœurs et vos sens en Christ Jésus).

En 1913, il avait rappelé à ses paroissiens l'importance de l'union à Jésus par l'action - un des leitmotivs de sa prédication entre 1899 et 1912 - et conclu son sermon par des accents missionnaires: il avait invité ses auditeurs à rechercher, ensemble, la paix et l'union de leur "pauvre volonté humaine» à celle de Jésus, de sorte que leur richesse intérieure et leur joie de vivre croissent, et qu'ils montrent ainsi la voie au reste de l'humanité(11).

Il avait choisi ${ }^{(12)}$ ce texte en 1913, à une époque où l'on redoutait un conflit entre les grandes puissances européennes. Mais ce texte était également de mise à l'automne de 1918, après quatre ans d'un conflit sanglant qui n'était pas encore achevé. Certes, Schweitzer n'avait pas connu personnellement les combats, meurtriers, qui s'étaient déroulés notamment en Alsace, sa région natale. Mais son père, qui était resté à Gunsbach, non loin de la ligne de front, durant toute la guerre, l'avait tenu informé de la situation dans le village de son enfance ${ }^{(13)}$. Quant à sa mère Louise, qui avait désapprouvé son départ pour l'Afrique, elle avait été une victime de la guerre: renversée par le cheval d'un soldat allemand, elle était décédée sans revoir Albert ${ }^{(14)}$. Surtout, on peut imaginer que, dès son retour, Schweitzer n'a pas manqué de s'entretenir à propos de la guerre tant avec les habitants de la vallée de Munster que, plus tard, avec ses paroissiens de Saint-Nicolas. Et puis, lui-même avait pu constater les ravages causés par la guerre au Congo français:

«Ces glorificateurs de la guerre, écrivait-il avec indignation en 1917 après avoir lu un article de journal qui comptait la guerre en valeur au nombre des "nobles aspirations à la gloire"

10 Voir M. Arnold, Albert Schweitzer. Les années alsaciennes (note 2), p. 234 sq.

11 Voir A. Schweitzer, Predigten 1898-1948 (note 2), p. 1195.

12 À la différence de la plupart de ses collègues, qui prêchaient sur l'un des textes fixés pour le dimanche, en fonction de l'année liturgique, Schweitzer avait pour habitude de choisir librement le passage biblique à partir duquel il prêchait.

13 Voir Matthieu ARNOLD, "Quand Louis Schweitzer racontait la "Grande guerre" à son fils Albert», Revue d'Histoire et de Philosophie religieuses, 97 (2017), p. 265-271.

14 Voir L. Schweitzer, Journal (note 9), p. 188 sq. 
de l'être humain, la voient peut-être idéalisée en quelque sorte par l'enthousiasme ou le sentiment de légitime défense. Mais leur exaltation tomberait peut-être, s'ils faisaient une seule journée de marche par les sentiers de la forêt vierge, sur l'un des théâtres de la guerre en Afrique. Ils rencontreraient à chaque instant les cadavres des porteurs qui, dans la solitude, ont succombé sous leur fardeau. Ces victimes innocentes, parties sans enthousiasme, dans l'obscurité et le silence de la forêt vierge, leur feraient comprendre ce qu'est réellement la guerre» ${ }^{(15)}$.

Dans sa prédication du 13 octobre $1918^{(16)}$, Schweitzer constate avec douleur les changements qui, depuis son départ, se sont produits dans sa communauté de Saint-Nicolas :

«Parmi les jeunes garçons qui, du haut de la tribune, entonnaient des chorals d'une voix claire, nombreux sont ceux qui sont morts adultes et reposent sur une terre lointaine. Tous, vous avez connu des privations sévères et avez perdu des êtres qui vous étaient chers. Nombre d'entre vous ont vu s'écrouler ce qu'ils avaient acquis par leur travail. D'autres ont dû donner leurs fils alors qu'ils comptaient sur eux pour leurs vieux jours. D'autres encore ont peiné afin d'assurer l'avenir de leurs enfants, et ces derniers ne sont plus là » ${ }^{(17)}$.

Dans ces circonstances, comment traiter le thème de sa prédication et parler de la «paix de Dieu»? Comment passer de la douleur au recueillement apaisé? Schweitzer refuse tout discours théologique convenu qui consisterait à tenir la «volonté de Dieu» (Gottes Wille) pour un principe explicatif universel qui interdise toute révolte voire toute interrogation humaines. Certes, fait-il observer, lorsqu'on considère les événements avec quelque distance, souvent on comprend ce qui, sur le moment, paraissait obscur. Toutefois, dans la vie des individus comme dans celle des peuples et de l'humanité, bien des choses demeurent dépourvues de sens, dans lesquelles le mal ne se dissout pas simplement dans le bien:

"Celui qui veut expliquer pourquoi une mère doit donner son fils unique, pourquoi un ami trahit son ami et pourquoi les phrases creuses triomphent de la vérité sombrera dans la confusion ${ }^{(18)}$.

Tout ce que Schweitzer peut dire, c'est que la volonté de Dieu est orientée vers un but spirituel ${ }^{(19)}$. Il prend ainsi le contrepied des prédications de guerre, qui liaient cette volonté à des triomphes militaires. Il ne cherche pas non plus à expliquer les événements, mais il se demande à quoi ils pourront servir: «Que vais-je faire d'eux? " ${ }^{(20)}$. En d'autres termes, non pas: «Pourquoi se sont-ils passés?», mais : "Quelle leçon spirituelle puis-je en tirer?» Pour Schweitzer, les événements doivent conduire à ce que «nous autres, êtres humains, les peuples, l'humanité deviennent toujours plus parfaits [vollkommener]». C'est en cherchant la pacification (Befriedigung) que nous parviendrons à la paix

15 Albert Schweitzer, À l'orée de la forêt vierge. Récits et réflexions d'un médecin en Afrique équatoriale française (1921), Paris, Albin Michel, 1995, p. 209.

16 Le manuscrit autographe de ce sermon a été présenté au public lors de l'exposition Schweitzer à la Bibliothèque nationale et universitaire (Strasbourg). Voir le catalogue Albert Schweitzer. Entre les lignes, sous la dir. de Benoît Wirrmann et Jean-Paul Sorg, Strasbourg, 2015, p. 109.

17 A. Schweitzer, Predigten 1898-1948 (note 2), p. 1199.

18 Voir ibid., p. 1199.

19 Voir ibid., p. 1200.

20 Ibid., p. 1200. 
(Friede) de Dieu ${ }^{(21)}$. Ce discours à la première personne du pluriel, avec un «nous» qui englobe l'humanité tout entière, est remarquable. Certes, Schweitzer évoque en passant l'avenir spirituel du peuple allemand («unser Volk» ${ }^{(22)}$ ). Mais il parle, bien plus longuement, des «autres peuples» et de «tous les peuples» ${ }^{(23)}$. Le souci pour le peuple allemand ne s'oppose pas à celui pour l'humanité tout entière, bien au contraire:

«Et de même que, pour trouver la paix, il nous faut penser à l'avenir spirituel de notre peuple, il nous faut songer également à celui de l'humanité. Il faut qu'advienne une humanité au sein de laquelle les peuples soient unis par des visées spirituelles et qu'ils aspirent à ce qu'il y a de plus élevé ici bas » ${ }^{(24)}$.

Pour que cette humanité (Menschheit) ait un avenir, il faut, explique le prédicateur, abandonner les faux idéaux et passer de l'absence d'humanité (Nichthumanität) à l'humanité (Humanität). Et Schweitzer de réintroduire - en dépit du "chaos dans lequel se débat aujourd'hui l'humanité» - le thème du Royaume de Dieu, leitmotiv de sa prédication d'avant $1914^{(25)}$. Il s'agit de l'idée que, petit à petit, des êtres humains, toujours plus nombreux, seront gagnés par l'esprit de Jésus et vivront conformément à son message:

«Ce que nous pensons, nous autres gens religieux, n’a rien à voir avec [la propagande], mais se rapporte à ce que notre Seigneur exprimait par les mots "Royaume de Dieu". [...] Sans doute les événements qui se sont accumulés entre les êtres humains ajournent-ils actuellement ce qui ressemble au Royaume de Dieu. Il nous faut écarter des obstacles qui n'existaient pas auparavant. Mais lorsque, dans le cœur de personnes nombreuses, se lèvera l'état d'esprit qui doit venir, alors tous ces obstacles seront surmontés. Lorsque l'Esprit se manifeste avec pureté et puissance, rien ne peut lui résister » ${ }^{(26)}$.

C'est pourquoi Schweitzer peut conclure sa prédication avec des accents optimistes, même s'il sait que l'avènement d'une humanité meilleure prendra un certain temps:

«Nous avons enterré des hommes et des espérances plus que nulle autre [génération] dans l'histoire. Mais de la destruction par laquelle il nous a fallu passer nous voulons sauver la foi en l'avenir de l'humanité [Menschheit] comme l'idéal le plus précieux et, dans cette époque nouvelle, le transmettre aux générations futures ${ }^{(27)}$. Le soleil de l'espérance ne luit pas encore sur nos chemins. Pour l'instant, la nuit se prolonge, et notre génération ne connaîtra plus l'aube du jour nouveau. Mais, si nous sauvons la foi en ce qui doit venir, alors la lumière des étoiles éclairera notre chemin. Viens, paix de Dieu, remplis nos cœurs, viens à notre secours! » ${ }^{(28)}$.

21 Voir ibid.

22 Ibid., p. 1201 sq.

23 Voir ibid., p. 1201 sq.

24 Ibid., p. 1202.

25 Voir Albert Schweitzer, L'Esprit et le Royaume, traduit de l'allemand et présenté par Jean-Paul Sorg, s.1., Arfuyen, 2015; Matthieu Arnold, Albert Schweitzer. La compassion et la raison, Lyon, Olivétan, 2015.

A. Schweitzer, Predigten 1898-1948 (note 2), p. 1202.

Ibid., p. 1202 sq. 
Après avoir, le 3 novembre 1918, abordé des questions plus terre à terre, les soucis quotidiens de ses paroissiens, et avoir appelé ces derniers à la solidarité( ${ }^{(29)}$, Schweitzer revient, le 24 novembre 1918, à la question de l'avenir de l'humanité.

Trois jours plus tôt, les soldats allemands ont quitté Strasbourg et les troupes françaises ont été accueillies en triomphe. Désormais, maints Strasbourgeois donnent libre cours à leurs sentiments de revanche, voire de haine, à l'égard des "Vieux-Allemands". C'est donc dans une atmosphère pesante que Schweitzer est amené à prendre la parole, devant un auditoire qui, sans doute, mêle Altdeutschen et Alsaciens de souche. Sur le plan de l'année liturgique, le culte du 24 novembre est consacré au «souvenir des défunts »: lors de ce dernier dimanche de l'année ecclésiastique, qui précède le temps de l'Avent, on a coutume, dans l'Église de la Confession d'Augsbourg, de faire mémoire des défunts de l'année qui s'achève; des représentants de leurs familles sont présents, et l'on peut donc penser qu'en l'église Saint-Nicolas, l'auditoire est nombreux ce matin-là.

Schweitzer commence sa prédication de manière abrupte, sans enjoliver les circonstances effroyables dans lesquelles on meurt durant la guerre:

«Pour la cinquième fois [depuis 1914], alors que l'automne s'apprête à céder la place à l'hiver, il nous faut faire mémoire non seulement de ceux qui sont morts emportés par l'âge, la maladie ou un accident, mais aussi de ceux qui, dans une guerre meurtrière, sont tombés frappés par la main d'hommes. Comment sont-ils morts? Un coup de feu a traversé leur corps et ils se sont vidés de leur sang; durant des jours, ils se sont consumés dans les gémissements, accrochés aux fils de fer barbelés, sans que personne ne puisse leur porter secours; dans la nuit, ils sont morts de froid sur une terre gelée; des explosions les ont ensevelis ou les ont projetés dans les airs après les avoir déchiquetés; en bouillonnant, l'eau a entraîné par le fond le bateau sur lequel ils se trouvaient; ils se sont battus contre les vagues jusqu'à l'épuisement ou, enfermés dans la cale, se sont arc-boutés contre ses parois, apeurés et impuissants. Quant à ceux qui ne sont pas morts sur terre ou dans l'eau, ils s'en sont rentrés, après avoir souffert à l'hôpital militaire tous les tourments durant des semaines et des mois, pour être contraints de lutter avec la vie et l'existence d'un estropié» ${ }^{(30)}$.

Toutefois, ces souffrances endurées par des hommes des mains d'autres hommes appartiennent au passé. Il s'agit maintenant de faire mémoire d'eux. De quelle manière? «Je crois, poursuit Schweitzer, qu'il nous faut - nous autres hommes [Menschen] de tous les peuples - leur promettre quelque chose» ${ }^{(31)}$. Quel est le contenu de cette promesse?

"Que leur mort n’a pas été vaine. Ils se sont sacrifiés dans tous les pays, chacun pour protéger son peuple contre les atrocités de la guerre et pour lui préserver la paix. Et il faut que chaque peuple remercie ses morts pour cela. Dans les pays auxquels a été accordée la victoire, on exprime la signification de leur mort par les cris d'allégresse qui bruissent au-dessus des tombeaux. Dans [les pays] qui ont été vaincus, on songe à eux avec douleur. Ce sont les circonstances extérieures qui ont décidé que pour les uns la mort scellerait la victoire, et pour les autres elle ne serait pas couronnée de succès. Mais telle n'est pas la signification ultime de leur mort. Désormais, alors que nous regardons la guerre comme quelque chose de passé, ceux qui ont été sacrifiés se tiennent comme une troupe au sein de 
laquelle il n'y a plus de différence de race ni de nation, comme des êtres humains qui sont unis dans la douleur et la souffrance, et qui exigent de nous quelque chose ${ }^{(32)}$.

Avant d'exposer le contenu de cette exigence, on relèvera que Schweitzer parle des «circonstances extérieures» qui ont décidé de la victoire ou de l'échec. Schweitzer se distingue de ses collègues qui liaient victoire et cause juste, voire qui associaient Dieu au triomphe du camp de la «justice», de la "vérité» et du «bien». Deux jours après Schweitzer, son ancien collègue de Saint-Nicolas, Charles Théodore Gérold, prêchera dans le cadre d'un «Te Deum » - culte d'action de grâces destiné à remercier Dieu pour la victoire de la France - en l'église protestante du Temple Neuf. À la différence de la prédication de Schweitzer, le sermon de Gérold n'a pas été conservé, mais le résumé qu'en donne la brochure destinée à immortaliser la célébration ${ }^{(33)}$ nous renseigne sur son contenu: après être monté en chaire, le pasteur Gérold, est-il écrit, rend hommage «à nos frères français, ces vaillants soldats qui, au premier appel de la patrie, ont quitté leurs familles, leurs travaux, leurs projets et leur espérance, et ont marché d'un seul cœur pour la délivrance des frères captifs et la défense de la patrie ${ }^{(34)}$.

La prédication de Schweitzer, quant à elle, fait mémoire de toutes les victimes de la "Grande guerre», quelle que soit leur nationalité. Surtout, son contenu la rapproche d'une confession du péché plus que d'un hommage aux disparus:

«C'est de notre faute [Schuld] qu'ils sont morts. Avec quelle légèreté [avant la guerre], on songeait, dans tous les peuples, au bien-être et aux maux de l'individu [des einzelnen Menschen]. On avait trop peu de considération pour la vie humaine, cette valeur mystérieuse et irremplaçable» ${ }^{(35)}$.

Aussi faut-il désormais accorder à la vie bien plus d'importance qu'on ne l'a fait jusqu'alors:

«Nos enfants devront puiser dans notre expérience et garder tout au long de leur vie, comme un héritage qui leur a été légué, la conviction que le commandement "Tu ne tueras point" a une valeur beaucoup plus fondamentale que nos parents et nous-mêmes ne le pensions. [...] Que le respect de la vie et de la souffrance humaines - même à l'égard des plus humbles et des plus obscurs d'entre les hommes - soit désormais la loi d'airain qui régisse le monde! ${ }^{(36)}$.

«Respect de la vie» (Ehrfurcht vor dem Leben) : l'expression est lancée. C'est la première fois que ces termes, que Schweitzer avait prononcés dans un de ses derniers cours à Strasbourg en février 1912, puis «redécouverts» en Afrique, apparaissent dans un de ses sermons ${ }^{(37)}$. Schweitzer s'attache à réconcilier les êtres humains entre eux, à appeler

32 Ibid.

33 En souvenir des services religieux qui ont été célébrés au Temple-Neuf de Strasbourg le 26 novembre 1918, pour fêter l'entrée des troupes dans notre ville..., Strasbourg, Imprimerie Alsacienne, 1919 (BNU Strasbourg: M.702.651). Ibid., p. 4.

35 A. Schweitzer, Predigten 1898-1948 (note 2), p. 1209 sq.

36 Ibid., p. 1210.

37 Voir Matthieu Arnold, «Le respect de la vie dans les sermons d'Albert Schweitzer, avant et après la fin de la Première Guerre mondiale (1918-1919)", in: Matthieu ARnOLD (dir.), Albert Schweitzer et le respect de la vie, Strasbourg, Association de la Faculté de Théologie protestante, 2018, p. 101-115. 
chacun d'entre eux à respecter la vie humaine; quelques mois plus tard, il plaidera, également du haut de la chaire, pour le respect de toute vie, la vie animale, voire végétale, comme la vie humaine. Appeler au respect de la vie était d'autant plus important que, durant la guerre, nombre de prédications protestantes s'étaient attachées à balayer les réticences que les soldats pouvaient éprouver devant le geste homicide: la vie de l'ennemi, leur objectait-on, ne valait rien, car il était l'incarnation du mal ${ }^{(38)}$.

Le sermon de Schweitzer est éloigné de tout manichéisme, de tout triomphalisme et de tout nationalisme. À tel point, sans doute, que si l'on ignore sa prédication d'avantguerre, qui tançait avec courage le nationalisme du Reich tel qu'il se manifestait aux colonies et ailleurs, l'on pourrait n'y entendre - à tort - que réserve voire hostilité à l'endroit des nouveaux maîtres de Strasbourg et de l'Alsace. Les morts de tous les pays, unis sans distinction dans le trépas, insiste-t-il, ont une leçon à donner aux vivants:

«Nous sommes appelés à faire le pas que jusqu'à présent l'humanité ne pouvait pas accomplir. Nous ne pouvons pas faire autrement, car les défunts nous y aident et nous y contraignent. "Il y aura encore plus de souffrance, de cris et de douleur, car le [monde] premier est passé." Ce qui est premier, c'est le monde dans lequel il n'y avait pas de respect pour la vie humaine, monde qui s'est détaché lui-même de la loi de l'amour divin pour se placer sous le pouvoir de la misère que des êtres humains peuvent causer à leurs semblables. Ce qui est second, l'autre monde, c'est le Royaume de Dieu, pour lequel Jésus, pour lequel des millions [de personnes] sont mortes durant ces mois si nous ne rendons pas leur mort inutile, nous la génération [Geschlecht] qui entend tout en n'entendant pas et qui voit tout en ne voyant pas [Matthieu 13,13]» ${ }^{(39)}$.

Comme dans sa prédication du 13 octobre, Schweitzer se garde de raviver les antagonismes nationaux. Si, au début de son sermon, il a parlé, dans un registre proche des sermons de ses contemporains, des millions de personnes "sacrifiées", c'est pour exhorter ensuite ses auditeurs à œuvrer à l'avènement d'une humanité réconciliée qui, enfin, prenne au sérieux le commandement du «respect de la vie». Il juge cette tâche d'autant plus urgente que «ce que nous négligerons de faire, durant des siècles, aucune génération au monde ne pourra le rattraper» ${ }^{(40)}$.

Le sermon du 24 novembre 1918 prononcé par Schweitzer différait tant des discours prononcés en chaire à la même époque qu'il valut à son auteur d'être surveillé par la police française ${ }^{(41)}$.

\section{Triomphe sur l'ennemi, réveil du protestantisme et redressement de la France: trois sermons français à titre de comparaison}

Rares sont les sermons de l'automne 1918 qui nous ont été conservés. Toutefois, nous pouvons comparer les sermons de Schweitzer à une prédication prononcée le 9 décembre 1918, soit moins d'un mois après l'Armistice, par le pasteur Pierre Maury

38 Voir les travaux de W. Pressel et de K. HAmmer cités à la note 7. Pour la France, voir Laurent GAMBAвотто, Foi et Patrie. La prédication du protestantisme français pendant la Première Guerre mondiale, Genève, Labor et Fides, 1996.

39 A. Schweitzer, Predigten 1898-1948 (note 2), p. 1211.

40 Ibid., p. 1212.

41 Voir ibid., p. 1208, note 19, qui se réfère à un manuscrit de Gustave WoytT, neveu de Schweitzer: Die Polizeiberichte über Albert Schweitzer, 1919-1921. 
à Castres ${ }^{(42)}$. Ce sermon porte sur un verset de l'épître de Paul aux Romains: «Tenant compte du temps où nous sommes, l'heure est venue de vous réveiller de votre sommeil» (Romains 13,11).

Comme Schweitzer, Maury utilise l'image d'une aurore ou d'une aube nouvelle, mais il estime qu'elle est déjà là, après «quatre années dans l'ombre de la souffrance et de la mort». Comme Schweitzer également, il estime que l'heure présente a une importance capitale, voire qu'elle est «unique». Sans grande surprise, le thème du sacrifice - et de la mémoire qu'il faut en faire - apparaît dès le début de sa prédication:

«Pensez, mes frères, à tous nos morts, à ceux qui ont compris que le don de leur vie était quelque chose de grand, quelque chose qui appartenait non au domaine médiocre des sentiments quotidiens, mais au domaine grandiose des destinées mêmes de l'espèce.»

Toutefois, la référence aux morts ne revêt pas la même fonction que dans le sermon de Schweitzer. Pour Maury, par leur grandeur, ces défunts qui ont fait «don de leur vie» doivent inviter les vivants à ne pas sombrer dans la «mesquinerie», l'«égoïsme» et la médiocrité, auxquels ils ont succombé une fois brisé l'élan des nobles aspirations de 1914. Lorsque Maury parle de la «solidarité sanglante» entre tous ceux qui ont vécu cette guerre, il s'agit d'une solidarité entre tous les membres de la même nation, la France, et non pas d'une solidarité entre les peuples. En revanche, alors que la dimension œcuménique était absente des sermons de Schweitzer, le sermon de Maury comporte des accents œcuméniques remarquables:

«Il faut enfin, mes frères, avoir le courage de le reconnaître. Nous avons trop vécu dans les chapelles de nos piétés étroites dans une attitude à la fois peureuse et hautaine. Et nous, protestants, devons particulièrement nous accuser et nous humilier, portant peut-être l'hérédité d'anciennes persécutions, orgueilleux d'une foi laborieusement acquise, nous demeurons très loin et très seuls.»

Tandis que Schweitzer critiquait l'acquiescement de tous au nationalisme et le mépris de la vie qui en a résulté, Maury, dans son autocritique, dirige ses flèches principalement contre les ecclésiastiques protestants et contre les chrétiens:

«Et tous, après la surprise première, ont réalisé avec amertume que - de cet isolement spirituel - ils étaient responsables et que si l'Église n'est pas une lumière sur la route humaine, c'est que les chrétiens n'ont pas su la faire briller comme il fallait, ou qu'ils l'ont tenue si loin qu'elle n'était plus pour notre civilisation qu'un lumignon fumant, à demi éteint, sans puissance de chaleur ni de rayonnement!»

Dans une France plus marquée par le combat contre les Églises et le christianisme que dans l'Alsace de Schweitzer, Maury est sensible au «fossé» qui croît entre les protestants et le reste de la société, ainsi qu'à l'«indifférence» à l'égard de leur religion: pour lui, elle est plus mortifère pour le protestantisme que les «hostilités violentes» dont il a été victime dans le passé. Aussi estime-t-il que la survie de l'Église se joue à présent. Il faut que les protestants sortent de leur sommeil, sans quoi ce dernier glissera peu à peu vers la mort:

42 Texte à paraître dans la revue Foi \& Vie. Nous remercions Frédéric Chavel, maître de conférences à la Faculté libre de théologie de Paris (Institut protestant de théologie), de nous avoir communiqué ce sermon avant sa parution. Afin de ne pas déflorer la publication dans Foi \& Vie, nous paraphraserons ce texte et en donnerons seulement de brefs extraits. 
«Mais pour qui se pose en toute sincérité et toute angoisse le problème de l'avenir du protestantisme, pour nos pasteurs, nos conducteurs, les enseignants des enfants, un seul danger subsiste, une seule lacune, l'affaiblissement spirituel, la diminution de la vie, le sommeil qui précède la mort.»

C'est pourquoi, fidèle à son texte de prédication, Maury emploie l'image du « réveil», un terme chargé de sens pour ses auditeurs puisque, au cours de son histoire, le protestantisme avait déjà connu plusieurs mouvements de «réveil». Il invite ses ouailles à sortir de leur sommeil intellectuel, moral et social, et plus encore de leur «sommeil spirituel»- le sommeil le plus grave, «le sommeil dont nous mourons» - en faisant l'expérience personnelle de Dieu et du Christ.

Si, à la différence des sermons de Schweitzer, ces accents piétistes et cette perspective «ecclésiocentrée» dominent largement dans la prédication de Maury ${ }^{(43)}$, ce dernier ne néglige pas pour autant les bouleversements politiques qui sont en train de se produire: il évoque notamment la "Conférence de paix» que «l'humanité va rédiger demain». Il cite également des propos pleins d'espoir de J.-H. Rosny au sujet de la France:

«La France fière et lumineuse, la douce France où se rencontrent toutes les séductions de la terre, la France créatrice va renaître. Les hommes y retrouveront tout ce qui fait la beauté, la noblesse et l'orgueil de la vie» (L’Aube du Futur, Éditions Georges Crès, 1917, p. 99).

Tout aussi éclairante s'avère la comparaison entre les propos de Schweitzer et ceux que ses collègues Wilfred Monod et John Viénot, professeurs à la Faculté de théologie protestante de Paris ${ }^{(44)}$, ont tenus les 17 et 24 novembre $1918^{(45)}$.

Dans son discours du 17 novembre, "Après la victoire», Wilfred Monod donne aux événements récents une interprétation théologique: «Oui, le Fils de l'homme est revenu brusquement, en justicier, purifier le Temple» ${ }^{(46)}$. À le lire, le triomphe des «nations démocratiques» n'est rien d'autre qu'une «manifestation de l'Esprit du Serviteur de l'Éternel, le surnaturel “jusqu'auboutiste”, celui qui ne connaîtra jamais ni lassitude ni découragement “jusqu’à ce qu'il ait établi sur terre la justice” "(47). Il s'agit désormais pour la France de "gagner la paix», sans répondre aux vaincus par la violence, même si «les provinces délivrées ont eu à supporter des vexations intolérables et des crimes odieux» ${ }^{(48)}$, et, surtout, en menant désormais un combat moral pour le redressement de la nation:

«Préparons-nous, mes frères, à tirer loyalement toutes les conséquences morales du triomphe militaire. Soyons au premier rang pour combattre la tuberculose, la débauche,

43 Autre indice de cette perspective, le sermon se conclut par l'exhortation suivante: "Que nous-mêmes et que l'Église se réveille pour l'avènement glorieux du Christ Roi !»

44 Cette institution avait perdu 24 de ses étudiants. (Voir John VIÉnOT, «La délivrance», in: Victoire et délivrance - voir note suivante -, p. 53.)

45 Discours publiés dans Victoire et délivrance. Discours prononcés à l'Oratoire du Louvre et au Foyer de l'Âme les 10, 17 et 24 Novembre, $1^{\text {er }}$ et 8 Décembre 1918 par MM. Les Pasteurs J.-E. Roberty, Wilfred Monod et John ViÉnOT, Paris, Fischbacher, 1919. À maints égards, ces «discours» relèvent du genre de la prédication.

46 Ibid., p. 34.

47 Ibid., p. 36.

48 Ibid., p. 40. 
et cet infâme alcoolisme qui a failli ternir, de son haleine infecte, le radieux visage de la Victoire» ${ }^{(49)}$.

Et Wilfred Monod de lier ces injonctions morales aux serments solennels qu'il faut prêter tant aux jeunes gens «disparus dans la tourmente» qu'aux «survivants de la grande tribulation »:

«[...] jurons-leur solennellement que leur attente spirituelle ne sera point déçue, que leurs ferventes aspirations seront exaucées, enfin que nous servirons le Serviteur de l'Éternel sur le chemin de la justice intégrale, non seulement jusqu'à la victoire militaire, non seulement jusqu'à la victoire morale, mais encore jusqu'à la victoire mystérieuse de l'âme qui triomphe du péché» ${ }^{(50)}$.

Quant à John Viénot, il déclare, le 24 novembre - le jour où Schweitzer a prononcé son sermon sur les défunts -, que la délivrance «magnifique, infiniment joyeuse» est due non seulement "à nos morts", aux «combattants français et alliés" - «soldats magnifiques de la France envahie qui, pour libérer le sol national, pour résister à des attaques brutales ou perfides, [ont] su faire jaillir de nouveau en [eux] toutes les énergies cachées de la bonne race à laquelle [ils] appart[iennent]»-, mais encore «à Dieu même» ${ }^{(51)}$.

C'est le Dieu providentiel que Viénot exalte dans son allocution, celui-là même que prêchaient les pasteurs allemands au début du conflit en soutenant qu'il était le maître de la nature et de l'histoire! Voici en effet ce qu'il affirme:

«À qui, en dernière analyse, devons-nous notre délivrance [...] ? À qui, sinon à Celui à qui nous devons le mouvement, la vie et l'être, à Celui qui mène mystérieusement toutes choses vers un but; à qui, sinon au grand Capitaine de l'humanité montante, sinon à Celui qui inspire la patience dans l'épreuve, le courage dans le danger [...]; à qui, sinon à toi, ô Dieu, père de Jésus-Christ et père de l'humanité ? " ${ }^{(52)}$.

À la fin de son allocution, Viénot rapporte une conversation qu'il aurait eue l'année passée avec un pasteur de la Suisse allemande, lequel était «terriblement neutre»; aussi Viénot aurait-il passé sa soirée "à lui montrer que, particulièrement sur la question de l'Alsace, nous serions intraitables, que nous irions jusqu'au bout, parce que nous avions pour nous le droit et le bon droit» ${ }^{(53)}$. Après leur séparation, puis des mois de silence, le pasteur suisse lui a enfin donné raison dans une lettre récente. C'est pourquoi, Viénot peut clore son discours par une bénédiction dont, à la différence des prières qui concluent les sermons de Schweitzer, l'horizon reste strictement national:

«Ô Dieu des délivrances qui, à tous points de vue, fais lever l'heure de la justice pour notre patrie bien aimée, sois béni!» ${ }^{(54)}$.

49 Ibid., p. 42.

50 Ibid., p. 47 sq.

51 Ibid., p. 57.

52 Ibid., p. 60.

53 Ibid., p. 62.

54 Ibid., p. 63. Voir dans le même sens le discours de John ViÉnot du $1^{\text {er }}$ décembre 1918, "Action de grâce», qui énumère longuement les fautes de l’Allemagne, coupable châtié enfin par Dieu (p. 69-72), et qui se conclut de la sorte: «Vous, mes frères, qui voulez ne plus vous attacher désormais qu’à une 


\section{Conclusions}

Les prédications prononcées par Albert Schweitzer les 13 octobre et 24 novembre 1918 partagent plusieurs traits avec les sermons qui leur sont contemporains, voire avec les discours et les écrits de maints intellectuels à la fin de la Première Guerre mondiale puis au lendemain de ce conflit long et meurtrier. On y trouve en effet l'idée d'un moment favorable, d'une occasion à ne pas laisser échapper et à saisir au bénéfice d'un redressement; on y rencontre également le thème du serment que les vivants ont à prêter aux défunts, et tout particulièrement aux soldats tombés au front.

Toutefois, les différences nous semblent bien plus remarquables que ces parallèles. Ainsi Schweitzer, qui a refusé de mêler Dieu au conflit dans les réflexions qu'il a couchées sur le papier durant la guerre, ne l'associe-t-il pas davantage à la victoire dans ses sermons de l'automne 1918: ce sont les «circonstances extérieures »- et non pas la Providence - qui ont décidé de la victoire pour les uns, et de la défaite pour les autres. En ce qui concerne l'absence de triomphalisme de Schweitzer, sans doute s'explique-t-elle en partie par le fait que, dans sa région natale, l'Alsace, on était tombé dans un camp comme dans l'autre, ainsi que par le fait qu'Hélène, son épouse, était née de parents allemands. Mais elle s'explique surtout par le fait que, bien avant 1914, Schweitzer avait combattu la montée du nationalisme, dont - quitte à froisser Hélène il jugeait tous les grands États européens responsables, et avait plaidé pour l'avènement d'une humanité réconciliée. À l'automne de $1918^{(55)}$, il resta fidèle à ses convictions, et c'est ce qui lui permit de traiter de manière fort originale les thèmes qu'il semblait partager avec ses contemporains: ainsi, plutôt que d'appeler au redressement de la France ou de l'Allemagne, ou encore au réveil du protestantisme, il exhorta ses paroissiens à œuvrer pour que l'humanité tout entière, solidaire dans la chute, se relevât de concert; plutôt que de prôner un redressement moral de la patrie caractérisé par la lutte contre les vices, il fit d'une éthique nouvelle, le «respect de la vie» (Ehrfurcht vor dem Leben), le fondement de la civilisation qu'il appelait de ses vœux.

Dans l'immédiat après-guerre, marqué au coin de la revanche pour les uns et, bientôt, de la rancour liée aux conditions de paix pour les autres, les temps n’étaient hélas pas mûrs pour qu'un tel message fût entendu et diffusé largement. Mais que Schweitzer ait échoué à convaincre ses contemporains n'enlève rien au courage ni à la justesse de ses propos d'octobre-novembre 1918.

tâche immortelle, avec ce qui vous reste de force, ne dites plus rien, ne faites plus rien, ne donnez plus rien, sans évoquer le Dieu de Jésus-Christ et sans évoquer cette vision idéale d'amour fraternel, de libération et de justice qui se résume en ces trois mots: France, France, France !...» (p. 76).

55 Sur la situation en Alsace à cette époque, voir Marc LienHARD, «Le protestantisme alsacien en 1918 ", Positions luthériennes, 66 (2018), p. 243-251. 


\title{
Résumé
}

À la fin de la Première Guerre mondiale, Albert Schweitzer (1875-1965), parti à Lambaréné avec son épouse en 1913, puis interné avec elle, en tant que citoyens allemands, dans divers camps de prisonniers en France en 1917-1918, reprend son activité de prédicateur à la paroisse Saint-Nicolas de Strasbourg. Les sermons qu'il prononce le 13 octobre et le 24 novembre 1918 comportent des caractéristiques remarquables, comme le montre la comparaison avec des prédications de théologiens français de la même époque : dépourvus de tout triomphalisme et de tout nationalisme, ils appellent au redressement de l'humanité tout entière, grâce à l'éthique du "respect de la vie» (Ehrfurcht vor dem Leben).

\section{Zusammenfassung}

Nachdem Albert Schweitzer (1875-1965) im Jahr 1913 mit seiner Ehefrau nach Lambarene gereist und schließlich mit ihr als deutsche Staatsbürger von 1917 bis 1918 in verschiedenen Lagern in Frankreich interniert worden ist, nimmt er anschließend seine Tätigkeit als Prediger in der St. Nikolauskirche in Straßburg wieder auf. Seine Predigten vom 13. Oktober und vom 24. November zeichnen sich durch bemerkenswerte Eigenschaften aus, wie dies ein Vergleich mit Predigten französischer Theologen derselben Zeit deutlich macht: völlig frei von jeglichem Triumphalismus und Nationalismus rufen sie zur Wiederaufrichtung der ganzen Menschheit auf, im Namen der Ethik der „Ehrfurcht vor dem Leben".

\begin{abstract}
At the end of the First World War, Albert Schweitzer (1875-1965) resumed his post as preacher in St Nicholas' Church in Strasbourg, having left for Lambaréné (Gabon) with his wife in 1913, before being interned with her as German citizens in various prisoner camps in France in 1917-1918. The sermons he gave on 13 October and 24 November 1918 are remarkably distinct compared with sermons by French theologians in the same period: stripped of all triumphalism and all nationalism, Schweitzer's sermons are a call for a reconstruction of humanity in its entirety, thanks to the ethic of "respect for life" (Ehrfurcht vor dem Leben).
\end{abstract}

